\title{
COMPUTATIONAL STUDY OF FISH SCALE ARRAY
}

\author{
Saravanan $\mathbf{R}^{1}$, Gowtham $\mathbf{S}^{2}$, S.B Arjun Dhilip ${ }^{3}$, Harish $\mathbf{P}^{4}$, Henry $\mathbf{J}^{5}$ \\ ${ }^{1}$ Assistant Professor, Aeronautical Engineering, Hindusthan College of Engineering and Technology, \\ Coimbatore-32, Tamilnadu. \\ 2,3,4,5 UG-Aeronautical Engineering, Hindusthan College of Engineering and Technology, Coimbatore-32, Tamilnadu.
}

\begin{abstract}
Drag reduction is important in modern aviation because it improves both fuel consumption and aerodynamic performance. Better results in lift to drag ratio can be achieved by optimising the aerodynamic shape. The boundary layer separation can be prolonged up to a certain distance in the chord by creating turbulence over the wing surface under subsonic conditions. To improve the lift-to-drag ratio, additional components could be added to the wing's upper surface. Some of the components that can increase the lift-drag ratio of the wing include fish scales, inward dimples, and vortex generators. The fish scale array is designed, incorporated, and analysed on the upper surface of the NACA 4412 wing. To investigate the transition delay of the boundary layer on the section side of the airfoil, they were analysed for the angle of attack at $2^{0}, 4^{0}, 6^{0}, 8^{0}, 10^{0}, 12^{0}$. In comparison to a smooth airfoil, the lift to drag ratio also shows an improvement in performance.
\end{abstract}

Keywords: Drag reduction, Boundary layer, Lift-drag ratio, NACA 4412 wing, Smooth airfoil.

\section{INTRODUCTION :}

[1] Drag is a resisting force in fluid mechanics that acts against the relative motion of any object moving with respect to a surrounding fluid. For a laminar flow, drag force is proportional to velocity; for a turbulent flow, drag force is proportional to velocity squared. [2] When an aircraft is flying through the air, it encounters a variety of drag. Every drag has a source. The following categories are used to categorise different types of drag: Form drag, skin friction drag, lift-induced drag, and wave drag are examples of site drag. [3] Drag is determined by the fluid's properties as well as the size, shape, and speed of the object. The drag is one way to express the flow visualization in boundary layer conditions. Boundary layer separation is the most important factor affecting the generation of drag on an aircraft wing. The viscous forces near the wing surface are what cause boundary layer separation. [4] Under subsonic conditions, the flow over the wing's top surface is generally laminar. The flow attached to the wing's upper surface tends to detach as the angle of attack rises. Boundary layer separation is the term for this flow properties.[5] Vortices form in the flow aft of the boundary layer separation region, causing the aircraft to drag. The conversion of a laminar boundary layer to a turbulent boundary layer causes the boundary layer separation to be delayed, according to researchers. [6] The turbulent boundary layer has more momentum than the laminar boundary layer, allowing the flow to reattach to the surface more quickly. The mean kinetic energy per unit mass associated with eddies in a turbulent flow is referred to as turbulent kinetic energy. [7] Vortex generators and other drag-reducing components such as dimples, spoilers, and guide vanes are used in automobiles to improve vehicle performance. These extra components can also be used in planes to reduce wing drag.

\section{RESEARCH METHODLOGY :}

2.1 Fish Scale : Figure 1.2 Fish ScaleThe skin friction drag that occurs when a solid moving object interacts with a fluid was discussed in this analysis. The viscous drag in the boundary layer around the solid object causes it to generate this effect. A solid object moving through a fluid encounters a drag force as a result of skin friction, which restricts the object's forward motion. The performance of an object moving through a fluid flow can be improved by reducing skin friction. Aquatic organisms, such as fish, have special adaptations on their skin, such as scales, that allow them to swim faster through the sea by reducing drag. In general, a smooth surfaced object has less skin friction than a rough surfaced object. However, the smooth surface of an aircraft wing causes the formation of a laminar boundary layer, which detaches quickly from the wing's surface at higher angles of attack, causing excessive drag. According to studies, converting a laminar boundary layer to a turbulent boundary layer can cause the boundary layer separation to be delayed. Researchers are employing a variety of techniques to create the turbulent boundary layer. The fish scale has been discovered to aid in the formation of the turbulent boundary layer. The scale is a small, rigid plate that emerges from the fish's skin. 
Vol. 8, Issue 11, November 2021

DOI: $10.17148 /$ IARJSET.2021.81113

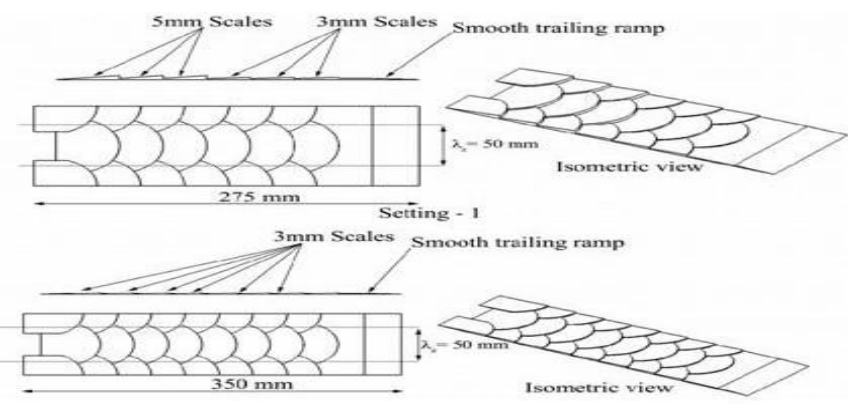

Figure 1. Fish Scale

\section{Fish scale pattern}

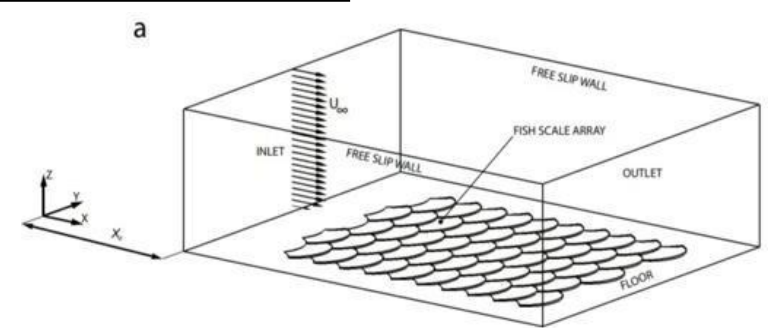

Figure 2. Modelling of Fish Scale array

\subsection{Fish Scale Pattern :}

The skin friction drag that occurs when a solid moving object interacts with a fluid was discussed in this analysis. The viscous drag in the boundary layer around the solid object causes it to generate this effect. A solid object moving through a fluid encounters a drag force as a result of skin friction, which restricts the object's forward motion. The performance of an object moving through a fluid flow can be improved by reducing skin friction. Aquatic organisms, such as fish, have special adaptations on their skin, such as scales, that allow them to swim faster through the sea by reducing drag.

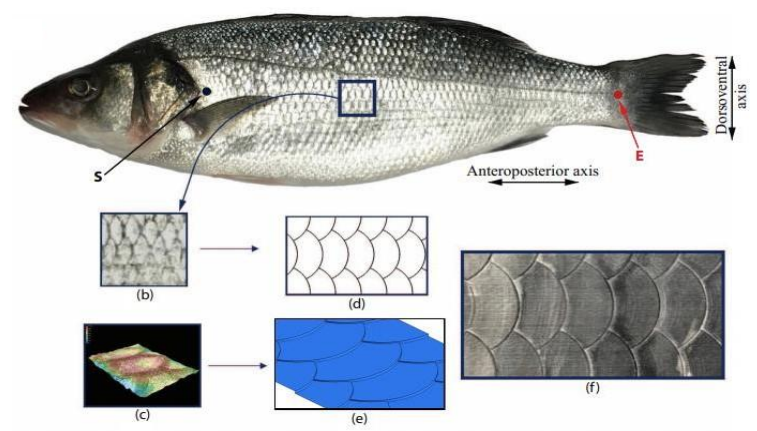

Figure 3. Fish Scale Pattern and Array

A smooth surfaced object has less skin friction than a rough surfaced object. However, the smooth surface of an aircraft wing causes the formation of a laminar boundary layer, which detaches quickly from the wing's surface at higher angles of attack, resulting in increased drag. According to studies, converting a laminar boundary layer to a turbulent boundary layer can delay the separation of the boundary layers. Researchers are using a variety of methods to create the turbulent boundary layer. Fish scales have been discovered to aid in the formation of turbulent boundary layers.

\subsection{Modelling :}

Concluded our discussion by designing an smooth airfoil of NACA 4412 in CATIA and also modelled our modified airfoil .With that knowledge in CATIA will do analysis in CFD analysis to know about the flow properties and then compare the NACA 4412 airfoil of different values of pressure, velocity, turbulence kinetic energy and stream flow to our modified airfoil. Then, find the values of Angle of attack as $2^{0}, 4^{0}, 6^{0}, 8^{0}, 10^{0}, 12^{0}$.

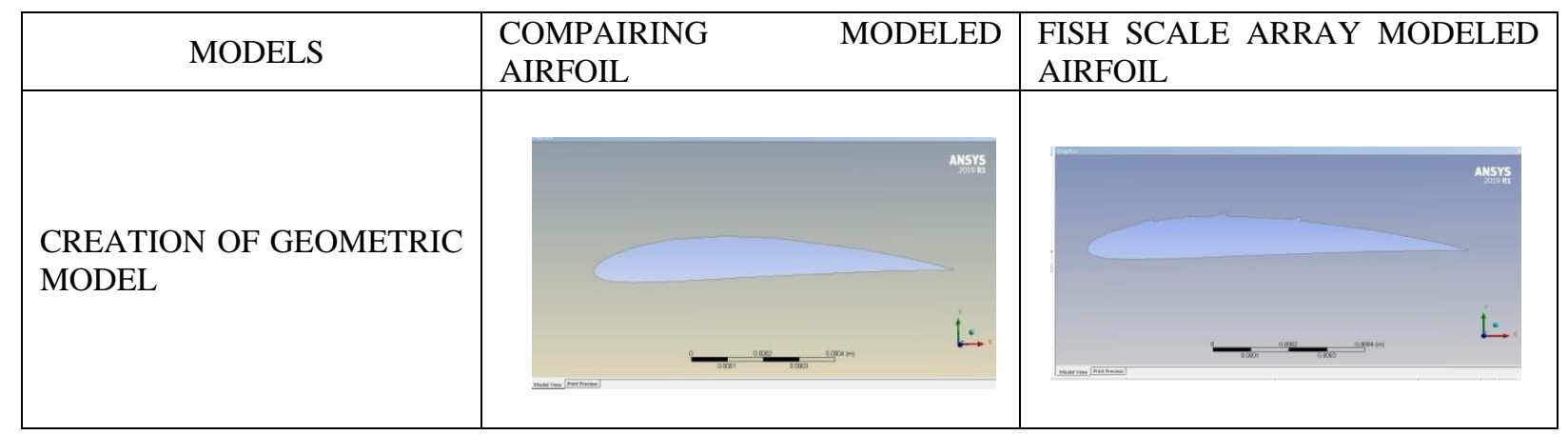


Vol. 8, Issue 11, November 2021

DOI: $10.17148 /$ IARJSET.2021.81113

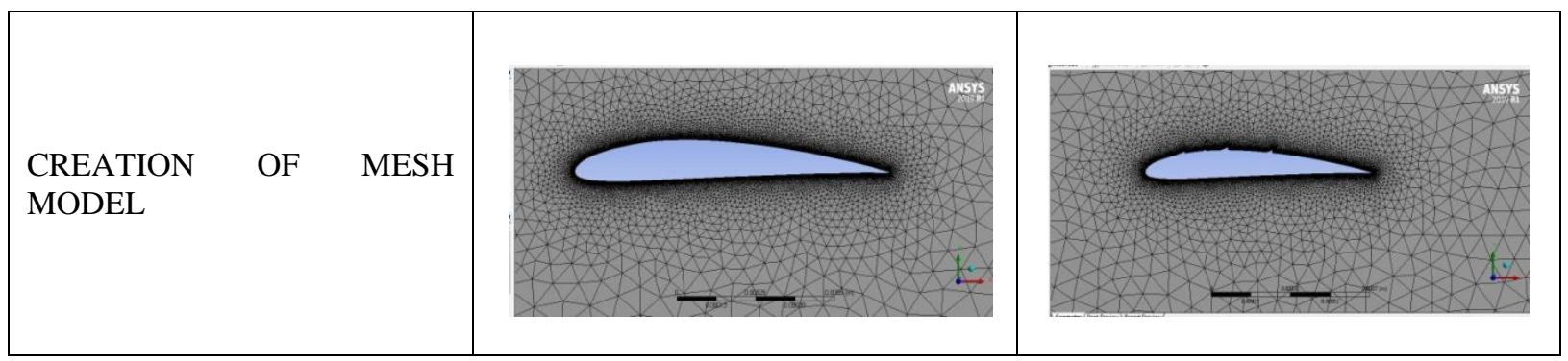

Fig.4. Comparing modelled airfoil and fish scale array airfoil (design and mesh)

The computational domain is sub divided into number of small element or cells called grid. There exists a two kind of grid formation known as structured and unstructured mesh. Then apply the unstructured mesh for our solution domain in ANSYS mesh for comparing NACA 4412 airfoil and Fish scale array airfoil.

\section{RESULT AND DISCUSSION :}

The modified wings are analyzed at six different angles of attack $\left(2^{\mathbf{0}}, 4^{\mathbf{0}}, 6^{\mathbf{0}}, 8^{\mathbf{0}}, 10^{\mathbf{0}}\right.$ and $12^{\mathbf{0}}$ degree $)$ and the magnitude of velocity is given. After the initial hybridization is done and the calculations are run. The plain wing without any drag reducing components is first analyzed at 12degree angle of attack and its CL and CD are obtained. It. is used to visualize all the contours. Blue represents the minimum value, green and red represents the maximum value.

\subsection{Pressure analysis of smooth and modified airfoil with different AOA}

At $2^{0}$ AoA for the surface modified surface of the regions there is no much effect in theflow variations comparing to the flow over normal profile aerofoil. At $4^{0}$ AoA for the surface modified surface of the regions there is no much effect in the flow variations comparing to the flow over normal profile aerofoil. If the modified profile is induced the flow gets resisted and there is the decrement of flow pressure which leads to decrement of aerodynamic efficiency. At $6^{0}$ AOA, the pressure flows smoothly over the smooth profile. However due to the fish scale surface at the upper section at the modified profile, there is an flow resistance which tends to increase in pressure in the suction side and the downstream flow. Like $2^{0}$ and $4^{0}$, this flow pattern has no such effects. At $8^{0}$ AoA the smooth profile, the flow near the trailing edge has low pressure since there is increased angle of attack. The pressure in modified surface shows that the flow leaving the trailing edge has low pressure compared to the smooth profile. The increased pressure is compared to the normal surface . At $10^{\circ} \mathrm{AoA}$, the pressure at the normal surface, there is the flow separation occurrence before theflow leaving the trailing edge. Due to the initial flow resistance at the suction surface there may be a increased chance of early flow separation which decreases the stall angle and decreases the $\mathrm{Cl}$. But at the modified surface, due to the near stall angle there is flow separation resistance due to the energized flow which tends to make the flow leaves at the trailing edge. After the stall angle, the pressure at $12^{0}$ AoA for modified profile shows that the perfect stall delay and flow separation. While this modified surface shows so such effect than other modified surface. This is due to the reenergizing of the vortex flow. Even after the stall angle, modified surface give delayed flow separation with decrement in the drag when comparing to other surfaces. 
International Advanced Research Journal in Science, Engineering and Technology

Vol. 8, Issue 11, November 2021

DOI: $10.17148 / I A R J S E T .2021 .81113$

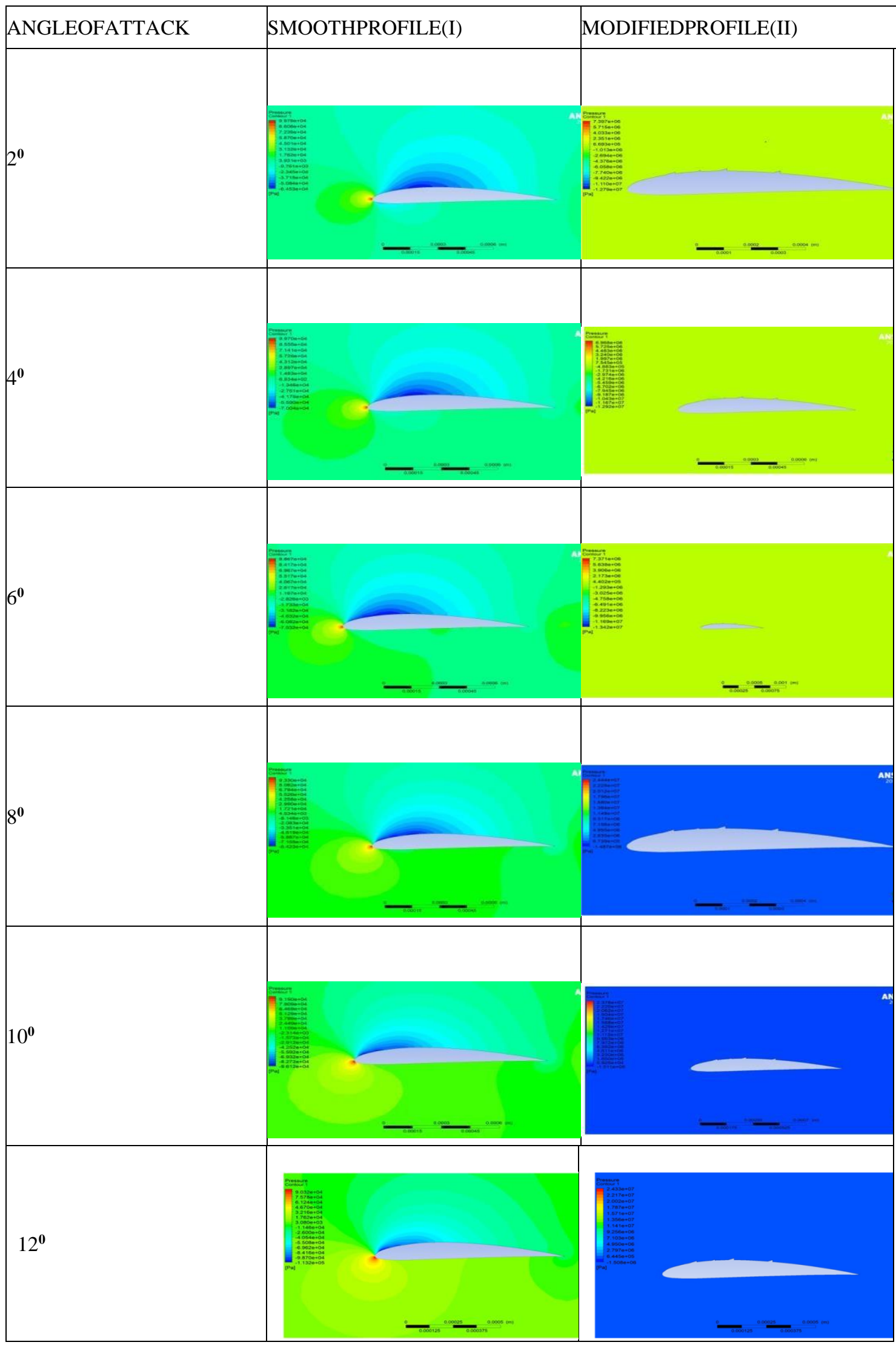

Fig 5. Comparing Pressure analysis of smooth and modified airfoil 
Vol. 8, Issue 11, November 2021

DOI: $10.17148 /$ IARJSET.2021.81113

\subsection{Velocity analysis of smooth and modified airfoil with different AOA}

At $2^{0}$ AoA for the surface modified surface of the regions there is no much effect in the flow variations comparing to the flow over normal profile aerofoil. At $4^{0}$ AoA for the surface modified surface of the regions there is no much effect in the flow variations comparing to the flow over normal profile aerofoil. If the modified profile is induced the flow gets resisted and there is the decrement of flow velocity which leads to decrement of aerodynamic efficiency. At $6^{0}$ AoA, the velocity flows smoothly over the smooth profile. However due to the fish scale surface at the upper section at the modified profile, there is an flow resistance which tends to increase in velocity in the suction side and the downstream flow. Like $2^{0}$ and $4^{\circ}$, this flow patternhas no such effects. At $8^{0}$ AoA the smooth profile, the flow near the trailing edge has low velocity since there is increased angle of attack. The velocity in modified surface shows that the flow leaving the trailing edge has low pressure compared to the smooth profile. The increased velocity is compared to the normal surface. At $10^{\circ} \mathrm{AoA}$, the velocity at the normal surface, there is the flow separation occurrence before the flow leaving the trailing edge. Due to the initial flow resistance at the suction surface there may be a increased chance of early flow separation which decreases the stall angle and decreases the $\mathrm{Cl}$. But at the modified surface, due to the near stall angle there is flow separation resistance due to the energized flow which tends to make the flow leaves at the trailing edge. After the stall angle, the velocity at $12{ }^{0} \mathrm{AoA}$ for modified profile shows that the perfect stall delay and flow separation. While this modified surface shows so such effect than other modified surface. This is due to the reenergizing of the vortex flow. Even after the stall angle, modified surface give delayed flow separation with decrement in the drag when comparing to other surfaces. 
International Advanced Research Journal in Science, Engineering and Technology

Vol. 8, Issue 11, November 2021

DOI: $10.17148 /$ IARJSET.2021.81113

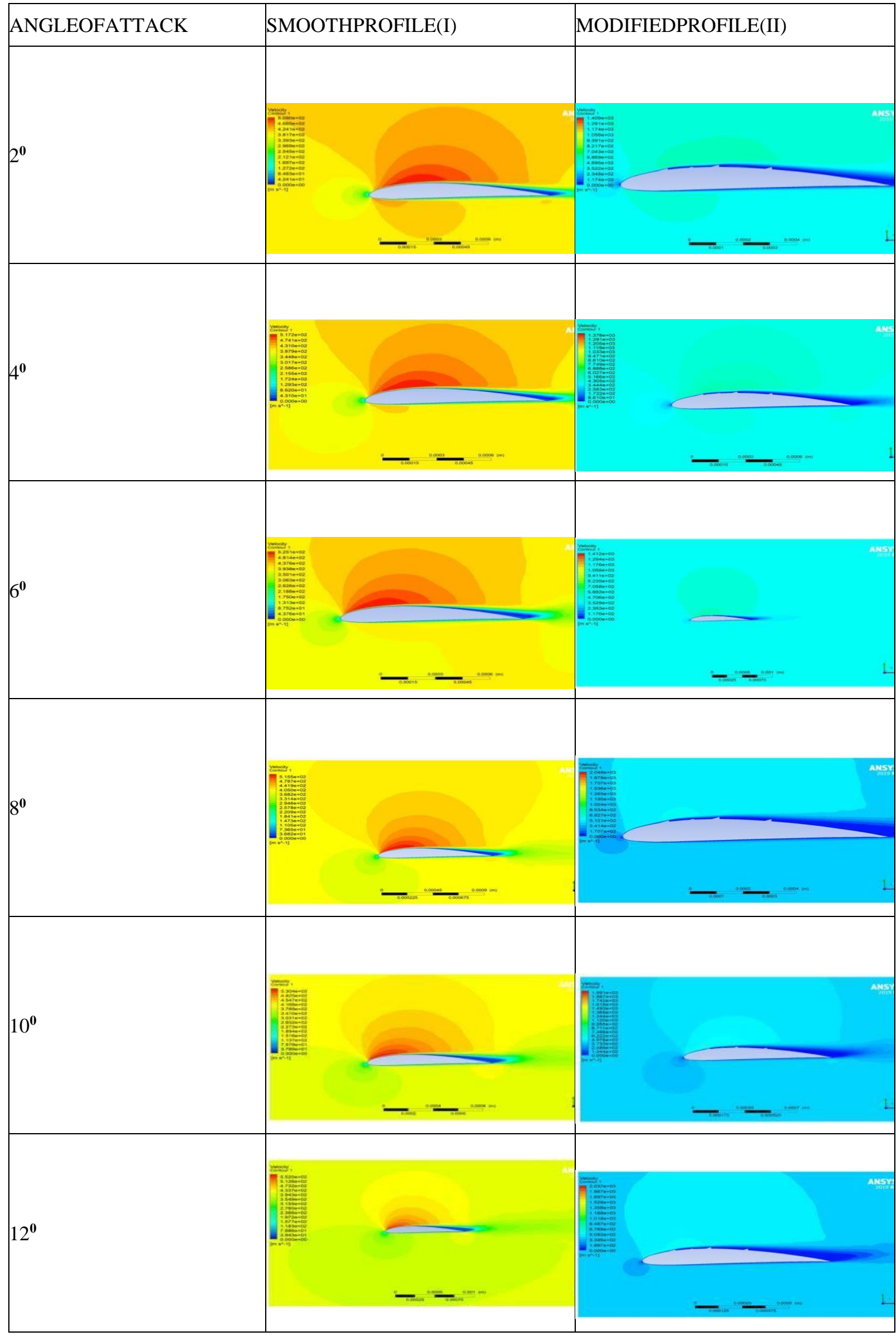

Fig 6. Comparing Velocity analysis of smooth and modified airfoil 
Vol. 8, Issue 11, November 2021

DOI: $10.17148 /$ IARJSET.2021.81113

\subsection{Turbulence Kinetic Energy analysis of smooth and modified airfoil with different AOA}

It is the normal profile occurs at the trailing edge with less amount $2^{0} \mathrm{AoA}$ due to modified profile induced at the upper surface the turbulent kinetic energy is created at the respective normal regions. Since the AoA is small the fish scale makes the decrement of the flow velocity which leads to slight decreasing the lift value. The turbulent kinetic energy at each modified section increases with increase in the AoA comparing to the $2^{0}$ AoA, the modified profile in $4^{0}$ AoA shows increase in the turbulent kinetic energy. Since the flow has lesser AoA, the modified profile at the surface shows noeffect. Turbulence kinetic energy at $6^{0} \mathrm{AoA}$ occurs at early stage of the flow leaving the trailing edge. At fish scale at upper surface, there is increment in the turbulent kinetic energy and it does not assist with the smooth profile turbulence kinetic energy. At modified upper surface, it assists the smooth profile. The turbulence kinetic energy created at the surface dissipates towards the trailing edge causing increase in the turbulent kinetic energy over surface. The flow leaving the trailing edge which is less energized compared to the surface. At $8{ }^{0}$ AoA the flow gets energized due to the swirling in the modified surface sections. The kinetic energy of the vortex increased with the increased turbulent kinetic energy. The flow gets re-energized which is going to delay the flow separation. Comparing all these above sections, the more aerodynamic performance is obtained Increase in turbulent kinetic energy followed to the trailing edge. From the given results, like the velocity streamlines the turbulent kinetic energy at the modified surface tends to reenergize the downstream flow in the suction surface. This makes delay in theflow separation. since there is flow separation starts early this makes the effect and tends to decrease in drag. From the above representation, at modified surface the turbulent kinetic energy tends to energizing the downstream flow thus delaying the flow separation. This decreases the drag compared to the smooth profile While surface 1 seems to have decrement in drag when compared to other angle of attacks. 
International Advanced Research Journal in Science, Engineering and Technology

Vol. 8, Issue 11, November 2021

DOI: 10.17148/IARJSET.2021.81113

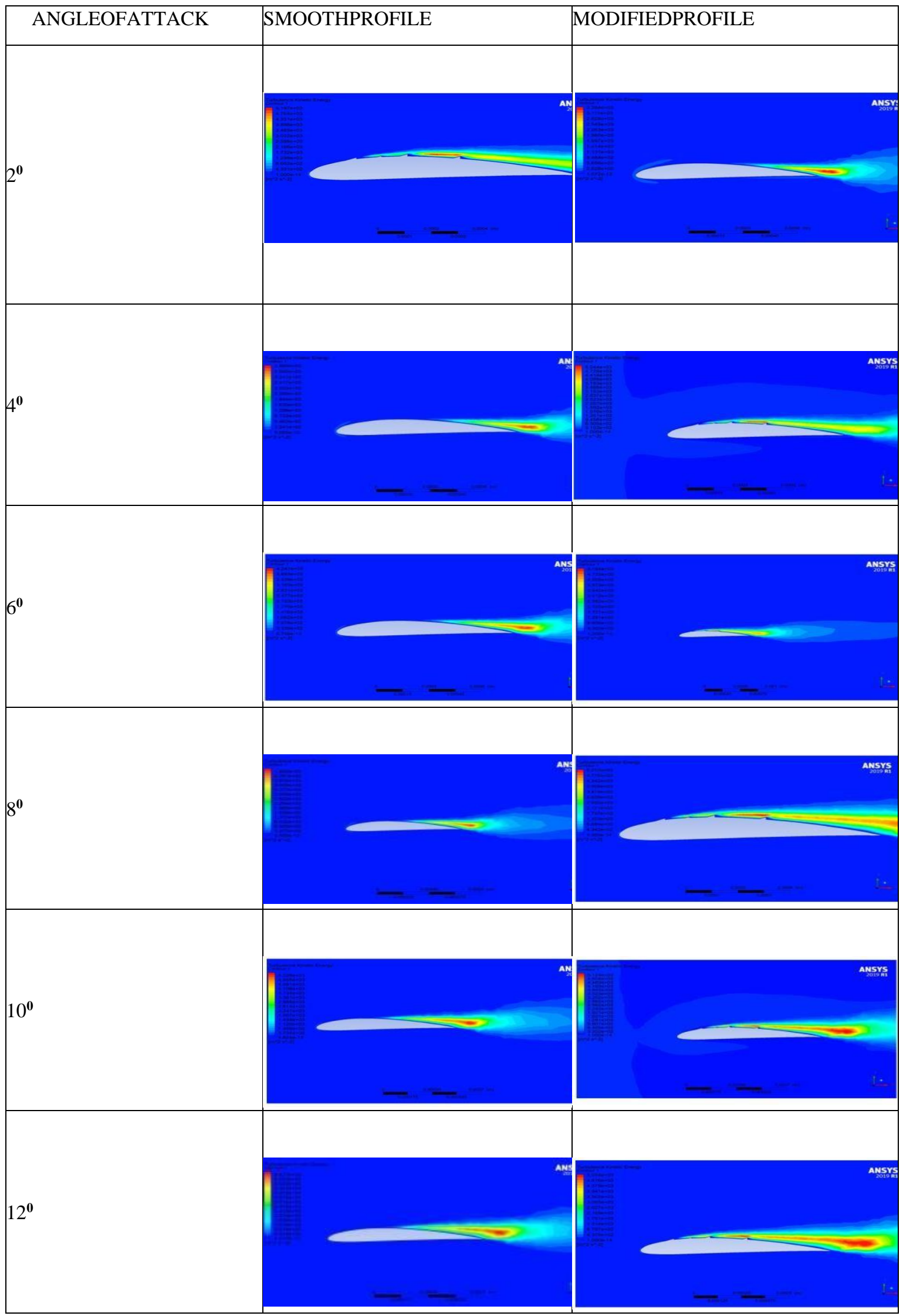

Fig 7. Comparing Turbulence Kinetic Energy analysis of smooth and modified airfoil 
Vol. 8, Issue 11, November 2021

DOI: 10.17148/IARJSET.2021.81113

\subsection{Stream Flow analysis of smooth and modified airfoil with different AOA}

Stream line flow in the normal profile occurs at the trailing edge with lesser amount at $2^{0}$ AoA due to modified profile induced at the upper surface the Stream line flow is created at the respective normal regions. Since the AoA is small the fish scale makes the decrement of the flow velocity which leads to slight decrement in the lift value. The stream line flow at each modified section increases with increase in the AoA comparing to the $2^{0} \mathrm{AoA}$, the modified profile in $4^{0}$ AoA shows increase in the stream line flow. Since the flowhas lesser AoA, the modified profile at the surface shows no effect. At $6^{0} \mathrm{AoA}$, the velocity stream line patterns flows smoothly over the smooth profile. However due to the fish scale surface at the upper section at the modified profile, there is an flow resistance which tends to increase in pressure in the suction side and the downstream flow. Like $2^{0}$ and $4^{0}$, this flow pattern has no such effects. At $8{ }^{\circ}$ AoA the flow gets energized due to the swirling in the modified surface sections. The kinetic energy of the vortex increased with the increased turbulent kinetic energy. The flow gets re-energized which is going to delay the flow separation. Comparing all these above sections, the more aerodynamic performance is obtained Increase in turbulent kinetic energy followed to the trailing edge. At $10^{0} \mathrm{AoA}$, the velocity stream line at the normal surface, there is the flow separation occurrence before the flow leaving the trailing edge. Due to the initial flow resistance at the suction surface there may be a increased chance of early flow separation which decreasesthe stall angle and decreases the $\mathrm{Cl}$. But at the modified surface, due to the near stall angle there is flow separation resistance due to the energized flow which tends to make the flow leaves at the trailing edge. After the stall angle, the velocity streamline flow at $12{ }^{0} \mathrm{AoA}$ for modified profile shows that the perfect stall delay and flow separation. While this modified surface shows so such effect than other modified surface. This is due to the re-energizing of the vortex flow. Even after the stall angle, modified surface give delayed flow separation with decrement in the drag when comparing to other surfaces. 
International Advanced Research Journal in Science, Engineering and Technology

Vol. 8, Issue 11, November 2021

DOI: $10.17148 /$ IARJSET.2021.81113

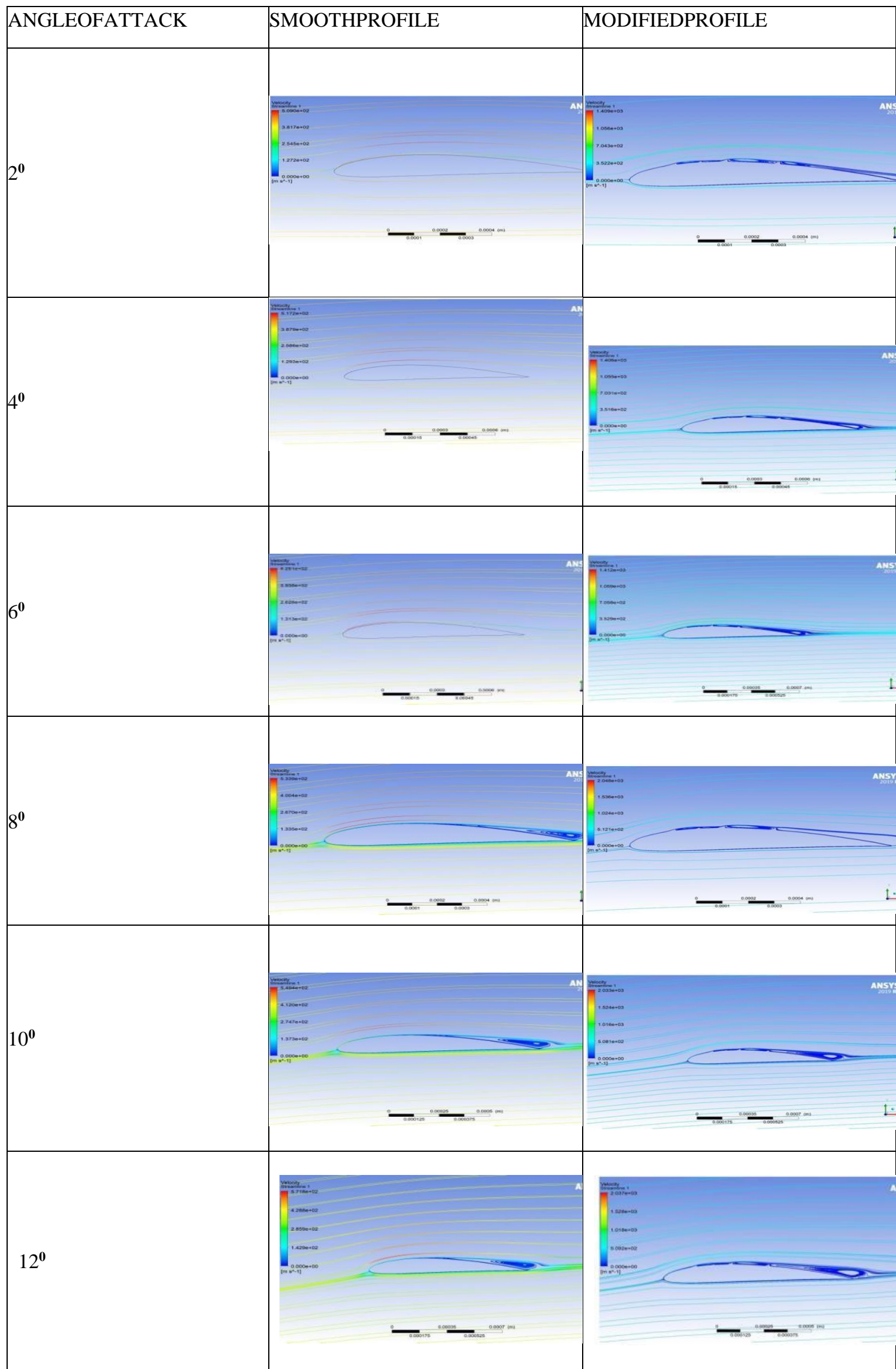

Fig 8. Comparing Stream Flow analysis of smooth and modified airfoil 
DOI: 10.17148/IARJSET.2021.81113

3.5. COMPARISON OF TABLE WITH NORMAL AND FISH SCALE AIRFOIL TABLE 1. NORMAL SURFACE AIRFOIL

\begin{tabular}{|l|l|l|l|}
\hline ANGLEOFATTACK & LIFT (N) & DRAG (N) & L/D \\
\hline 2 & 2.1375 & 0.7028 & 3.0414 \\
\hline 4 & 4.1627 & 0.7595 & 5.4808 \\
\hline 6 & 10.8918 & 0.9872 & 11.0330 \\
\hline 8 & 19.5636 & 1.0524 & 18.5895 \\
\hline 10 & 26.9471 & 1.1379 & 23.6814 \\
\hline 12 & 34.7648 & 1.2472 & 27.8742 \\
\hline
\end{tabular}

TABLE 2. FISH SCALE SURFACE AIRFOIL

\begin{tabular}{|l|l|l|l|}
\hline $\begin{array}{l}\text { ANGLEOF } \\
\text { ATTACK }\end{array}$ & LIFT (N) & DRAG (N) & L/D \\
\hline 2 & 2.3577 & 0.7861 & 3.9989 \\
\hline 4 & 5.2706 & 0.8623 & 6.1121 \\
\hline 6 & 13.4326 & 1.0171 & 13.2069 \\
\hline 8 & 24.5896 & 1.1164 & 22.0241 \\
\hline 10 & 31.5506 & 1.2119 & 27.0329 \\
\hline 12 & 39.7474 & 1.2965 & 34.0345 \\
\hline
\end{tabular}

\section{CONCLUSION :}

The coefficient of Lift and drag is calculated for this NACA 4412 series for the angle of attack $0^{\circ}$ to $12^{\circ}$. The coefficient of Lift/Drag ratio increases with increase in Angle of attack up to $8^{\circ}$. After $8^{\circ}$, Lift/Drag ratio decreases with increase in Angle of attack. On analyzing the airfoil at different angle of attacks, it has been obviously observed that lift to drag ratio drastically increases (under subsonic condition) when it is added up with certain aerodynamic components fish scales. These components have the ability togenerate vortices and the boundary layer flow changes from laminar to turbulent. Sothe unstable wave will generated in the smooth surface airfoil will be obtained and these wave will be not able to happen in fish surface airfoil. It results in delayed boundary layer flow separation and reduces the drag. The fish scale give better results compared to smooth surface airfoil. From the results it is evident that when fish scales are added on the upper surface of the airfoil it gives higher aerodynamic efficiency (L/D ratio increases), increases the stall angle, and gives better aircraft fuel economy. However, experimental studies might be needed to be performed. It is also necessary to determine the feasibility of generation of fish scales on airfoil. This concept cannot be applied toall airfoil profiles.

\section{REFERENCE :}

1. Oefner, J. \& Lauder, G. V. Te hydrodynamic function of shark skin and two bio mimetic applications. J. Exp. Biol. 215, 785-795 (2012).

2. Choi, H., Park, H., Sagong, W. \& Lee, S. Bio mimetic flow control based on morphological features of living creatures. Phys. Fluids 24,121302 (2012).

3. Pavlov, V., Riedeberger, D., Rist, U. \& Siebert, U. Analysis of the Relation Between Skin Morphology and Local Flow Condition s for a FastSwimming Dolphin, 239-253 (Springer, Berlin, 2012).

4. Gazzola, M., Argentina, M. \& Mahadevan, L. Scaling macroscopic aquatic locomotion. Nat. Phys. 10, 758-761 (2014).

5. Wen, L., Weaver, J. C. \& Lauder, G. V. Bio mimetic shark skin: design, fabrication and hydrodynamic function. J. Exp. Biol. 217, 1656-1666 (2014).

6. Dean, B. \& Bhushan, B. Shark-skin surfaces for fluid-drag reduction in turbulent flow: a review. Philos. Trans. R. Soc. A Math. Phys. Eng. Sci. 368, 4775-4806 (2010).

7. Fransson, J. H. M., Shahinfar, S., Sattarzadeh, S. S. \& Talamelli, A. Transition to turbulence delay using miniature vortex generatorsAphrodite. In Progress in Turbulence V (eds Talamelli, A. et al.) 71-74 (Springer, Cham, 2014)

8. Wainwright, D. K. \& Lauder, G. V. Mucus Matters: Te Slippery and Complex Surfaces of Fish

223-246 (Springer, Cham, 2017).

9. Wainwright, D. K., Lauder, G. V. \& Weaver, J. C. Imaging biological surface topographyin situ and in vivo. Methods Ecol. Evol. 8, 16261638 (2017).

10. $\mathrm{Wu}, \mathrm{L}$. et al. Experimental investigations on drag-reduction characteristics of bionic surfacewith water-trapping microstructures of fish scales. Sci. Rep. 8, 12186 (2018). 